\title{
Incidence of Esophageal Thermal Injury Using a Safety Protocol During Atrial Fibrillation Ablation
}

Anibal Pires Borges ${ }^{1, *}$, Guilherme Ferreira Gazzoni ${ }^{1}$, José Plutarco Gutierrez Yanez ${ }^{1}$, Karina

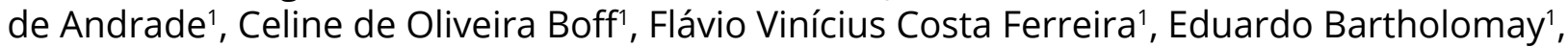
Álvaro Machado Rösler ${ }^{1}$, Fernando Antonio Lucchese ${ }^{1}$, Carlos Antonio Kalil ${ }^{1}$

\section{ORCID IDs}

Borges AP (D) https://orcid.org/0000-0002-3617-9589

Ferreira FVC (D) https://orcid.org/0000-0002-7186-707X

Gazzoni GF (D) https://orcid.org/0000-0001-5019-2475

Bartholomay E (D) https://orcid.org/0000-0003-0020-7944

YanezJPG (D) https://orcid.org/0000-0003-1523-0936

Rösler ÁM (D) https://orcid.org/0000-0003-4727-3367

Andrade K (D) https://orcid.org/0000-0002-6127-4617

Lucchese FA (D) https://orcid.org/0000-0001-9532-2384

Boff CO (iD) https://orcid.org/0000-0001-8575-4845

Kalil CA (D) https://orcid.org/0000-0003-0899-3062

\begin{abstract}
Objective: Catheter ablation has been a common procedure used for the management of atrial fibrillation (AF). Atrioesophagel fistula (AEF) is one of the most feared complications of AF ablation. Although it is a rare complication, severe esophageal thermal injury must be avoided. It is important to describe a safe method of preventing esophageal injuries without increasing AF recurrence. Methods: A retrospective cohort study of consecutive patients who underwent radiofrequency AF catheter ablation during 1 year-period was conducted. One hundred and four patients were enrolled divided in two groups: one with a maximum recorded esophageal temperature $(E T)<38^{\circ} \mathrm{C}$ and other with a maximum recorded $E T \geq 38^{\circ} \mathrm{C}$. The primary endpoint was detection of endoscopic esophageal lesions after AF ablation and the secondary endpoint was AF recurrence according to the maximum ET reached during the procedure. Results: The maximum ET was on average $37.3 \pm 1.0^{\circ} \mathrm{C}$. Only 4 (3.8\%) patients had esophageal lesion diagnosed by upper gastrointestinal endoscopy. There were no cases of esophageal perforation. The AF recurrence rate was $9.6 \%$ during the follow-up (10 patients, 3 from the ET max $<38{ }^{\circ} \mathrm{C}$ group and 7 from the ET max $\geq 38^{\circ} \mathrm{C}$ group; $\mathrm{p}=0.181$ ). The maximum ET was not associated with $\mathrm{AF}$ recurrence after catheter ablation $(O R=1.65,95 \% \mathrm{Cl}=0.84-3.24, p=0.14)$. Conclusions: $\mathrm{A}$ low incidence of esophageal injury after AF ablation with the use of a specific esophageal protection protocol was found. There was no esophageal perforation. The AF recurrence rate was similar to that described in the literature.
\end{abstract}

KEYWORDS: Atrial fibrillation; Catheter ablation; Esophageal injury; Esophageal temperature monitoring.

1. Hospital São Francisco - Santa Casa de Misericórdia - Porto Alegre/RS - Brazil.

*Corresponding author: anibalpiresborges@gmail.com

Received: Aug 11, 2020 | Accepted: Aug 18, 2020 


\section{INTRODUCTION}

Catheter ablation has increasingly been performed for the management of atrial fibrillation (AF) as a potentially curative approach $^{1}$. Since the discovery of the circumferential radiofrequency $(\mathrm{RF})$ ablation technique, with antral pulmonary vein isolation, there have been many improvements to make the procedure safer and effective. Although considered a minimally invasive procedure, some complications are reported.

The esophagus is located in close proximity to the posterior left atrium (LA), and thus ablation in that region may cause damage to the esophagus, resulting in complications such as periesophageal nerve injury, esophageal ulceration or atrioesophageal fistula $(\mathrm{AEF})^{2,3}$.

Atrioesophageal fistula is a rare but severe complication related to AF ablation. Esophageal injury (perforation or fistula) is described as occurring in approximately $0.5 \%$ of all $\mathrm{AF}$ ablation procedures ${ }^{4}$.

Whereas the exact mechanism of AEF is still unknown, esophageal thermal lesions as a direct result of ablation within the LA have been proposed to be the starting point of the cascade leading to esophageal perforation and AEF. In an attempt to explain this cascade, there are possible mechanisms of AEF formation: mucosal damage caused by thermal injury, gastroesophageal reflux potentially aggravating this damage and esophageal dysmotility preexisting or exacerbated by nerve injury during ablation or by general anesthesia 5 .

A Brazilian registry during 2003-2015 with 8,863 AF ablation procedures reported 10 cases of AEF (0.1\%). Seven patients died and 2 were discharged with neurological sequelae 6 .

There are many protocols for esophageal protection during AF ablation. Some use luminal esophageal temperature monitoring during procedure, but the benefit is not clear ${ }^{7}$. Esophageal active cooling by injection of ice-cold solution during RF energy delivery had beneficial effects as suggested by a meta-analysis of three studies ${ }^{8}$.

In the primum non nocere concept, trying to avoid esophageal damage during AF ablation procedures, Koneru et al. ${ }^{9}$ raised points to be discussed: distance of tissue wall and temperature rise, limiting or not ablation on the posterior wall, what power and application time, anesthetic method, the importance of mechanical displacement of the esophagus and the role of endoscopy in detecting incipient esophageal lesions. Due to these complications, it is necessary to describe a safe method of preventing esophageal injuries without increasing the recurrence of AF.

\section{METHODS}

A retrospective cohort study of consecutive patients who underwent radiofrequency AF catheter ablation was conducted during 1 year-period (between February 2019 and February 2020).

\section{Patient selection}

Patients were included in the study if they were at least 18-years-old, had symptomatic paroxysmal, persistent or longstanding persistent $\mathrm{AF}$ and were referred for RF catheter ablation. The patient's medical history, physical-examination findings and use of medications were recorded.

Patients in whom it was not possible to perform upper gastrointestinal endoscopy after the procedure and those whose ablation technique was cryoablation were excluded from this study.

\section{Ablation procedure}

For the ablation, contact-force sensing catheters ThermoCool SmartTouch NaviStar (Biosense Webster) or TactiCath (St.Jude Medical) were used depending on the chosen electroanatomical mapping system (CARTO or EnsiteNavix), with standard irrigation flow rates recommended by the catheter manufacturers. Contiguous ablations were made by dragging the catheter along the LA.

The aim of the ablation procedure was to achieve isolation of all pulmonary veins and to restore sinus rhythm in those who were in AF in the beginning of procedure. Additional RF applications were performed at roof and posterior wall ("box" lesion) in cases of persistent $\mathrm{AF}$ in which there was no reversion to sinus rhythm during pulmonary vein isolation. 


\section{Esophageal injury prevention protocol}

The esophageal injury prevention protocol consists of the following steps:

During procedure:

- Luminal esophageal temperature monitoring with multiple sensor catheter (Circa $\left.{ }^{\mathrm{TM}}\right)$ (Fig. 1). The alarm sounds when the esophageal temperature reaches $38^{\circ} \mathrm{C}$, so the applications are stopped until the temperature drops below $37^{\circ} \mathrm{C}$;

- Limiting energy delivered to the LA posterior wall (maximum $25 \mathrm{~W}$ );

- Mechanical displacement of esophagus with the transesophageal echocardiogram probe. It is used in special situations, only when pulmonary vein isolation is not achieved due to the rise in temperature at the application site;

- No esophageal cooling was performed during the study.

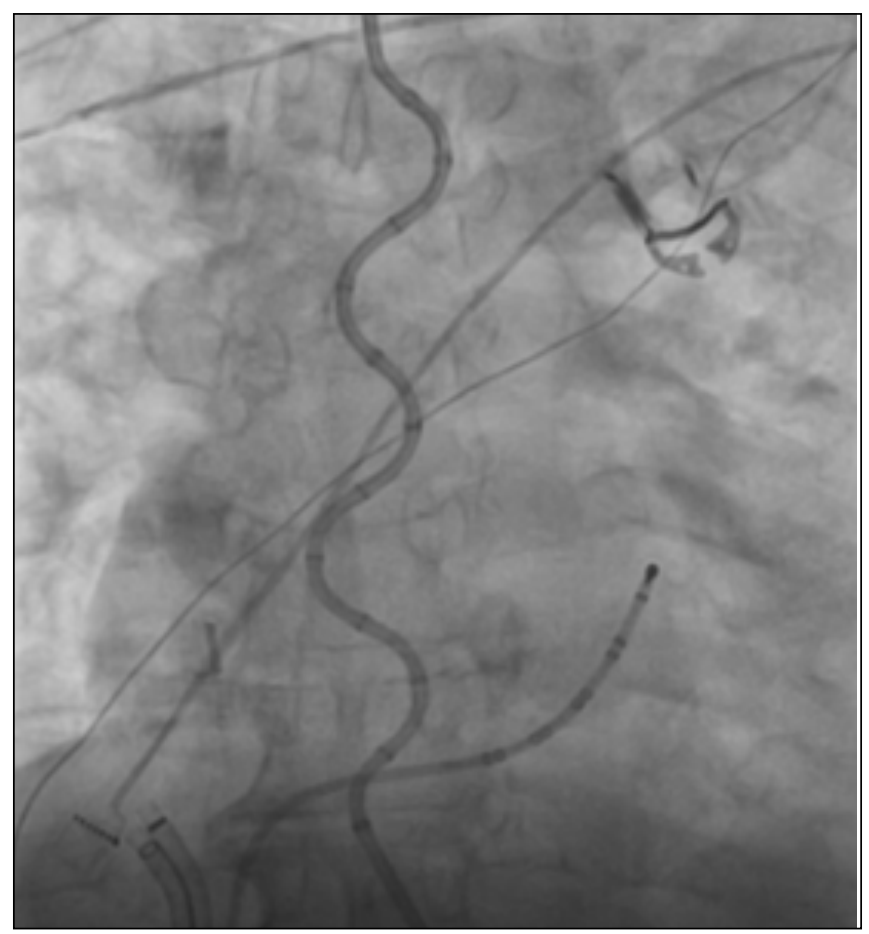

Figure 1. Luminal esophageal temperature monitoring with multiple sensor catheter (Circa).

After procedure:

- Postablation esophageal upper gastrointestinal endoscopy was performed, 24 to $48 \mathrm{~h}$ after the procedure;

- Pharmacologic gastric acid suppression. Patients undergo pharmacological therapy with proton pump inhibitor (PPI - omeprazole or pantoprazole, $40 \mathrm{mg}$ IV every $12 \mathrm{~h}$ ) and sucralfate ( $2 \mathrm{~g}$ orally every $6 \mathrm{~h}$ ). These doses are used until an endoscopy is performed;

- Liquid diet until endoscopy.

The next steps are taken according to the endoscopy result. If endoscopy is normal:

- Proton pump inhibitor orally until 30 days;

- Bland diet until discharge (usually on the 2nd day after the procedure). 
If endoscopically detected esophageal lesions (EDEL) are identified:

- Nothing by mouth or maintenance of liquid diet;

- Maintenance of PPI IV and sucralfate;

- Chest CT if the lesion is located at esophageal anterior wall;

- New endoscopy in $48 \mathrm{~h}$.

\section{Endpoints}

The primary endpoint was detection of endoscopic asymptomatic esophageal lesions after AF ablation using a prespecified protocol. The secondary endpoint was AF recurrence rate according to the maximum esophageal temperature (ET max) reached during the procedure. Atrial fibrillation recurrence was considered both symptomatic episodes and those documented by physical examination in follow-up visits or by complementary method (electrocardiogram, Holter etc.).

\section{Statistical analysis}

The data was stored in Microsoft Excel spreadsheets and analyzed using the Statistical Package for the Social Sciences (SPSS) software, version 21.0 (Armonk, NY, US: IBM Corp). Continuous variables were described as mean and standard deviation, or median and interquartile range, according to variable distribution. The evaluation of the normality of the quantitative variables was performed by calculating and observing the coefficients of asymmetry and kurtosis and by using the KolmogorovSmirnov test. Categorical variables were summarized as frequencies and percentages. Bivariate analysis was performed by the Spearman and Pearson correlation. In addition, a binary logistic regression was used to analyze the association of outcomes with possible predictors. Results were presented as odds ratio $(\mathrm{OR})$ and $95 \%$ confidence intervals $(\mathrm{CI})$, with $\mathrm{p}<0.05$ considered as statistically significant.

\section{Ethical aspects}

This study was performed in accordance with Brazilian standards and laws for studies on human beings and had ethical approval and authorization from the medical management of Hospital São Francisco. The study was entirely observational based in the medical routine and was conceived from the institution's permanent data record.

\section{RESULTS}

During the study period, 104 patients were enrolled, predominantly male (76.9\%), mean age of $57.7 \pm 12.1$ years. Eighty-four patients (80.8\%) had paroxysmal AF. The mean left ventricular ejection fraction (LVEF) was $61 \pm 11 \%$ and the mean anteroposterior diameter of the LA was $42 \pm 5 \mathrm{~mm}$. Almost $2 / 3$ of patients were using betablockers and/or antiarrhythmic drugs. The mean duration of the follow-up period was 12 months. The baseline characteristics of the patients are described in Table 1.

The objective of the procedure, isolation of all pulmonary veins, was achieved in 96 patients (92.3\%). The highest esophageal temperature (ET) was on average $37.3 \pm 1.0^{\circ} \mathrm{C}$.

Patients were allocated to two different groups according to the cutoff point of ET: one group with a maximum recorded ET less than $38^{\circ} \mathrm{C}$ (ET max $<38=56$ patients) and the other group with a higher recorded maximum ET or equal to $38^{\circ} \mathrm{C}$ (ET $\max =38$ patients).

Among all patients analyzed, only 4 (3.8\%) had esophageal lesion diagnosed by the upper gastrointestinal endoscopy, with 2 lesions in each of the groups described, respectively, 3.6 and 4.2\% (p 1.000) (Fig. 2 - esophageal lesion). All patients were treated conservatively according to the protocol described, with improvement in the lesion pattern in the control endoscopy (performed $48 \mathrm{~h}$ later). 


\begin{tabular}{|c|c|c|c|c|}
\hline Characteristics and outcomes & $\begin{array}{c}\text { TOTAL } \\
(n=104)\end{array}$ & $\begin{array}{c}\mathrm{ET}<38^{\circ} \mathrm{C} \\
(\mathrm{n}=56)\end{array}$ & $\begin{array}{c}\mathrm{ET} \geq 38^{\circ} \mathrm{C} \\
(\mathrm{n}=48)\end{array}$ & $p$ \\
\hline \multicolumn{5}{|c|}{ Demographic data } \\
\hline Male & 80 (76.9\%) & $45(80.4 \%)$ & $35(72.9 \%)$ & 0.369 \\
\hline Age (years) & $57.7 \pm 12.1$ & $57.3 \pm 12.5$ & $58.2 \pm 11.6$ & 0.698 \\
\hline \multicolumn{5}{|c|}{ Comorbidities } \\
\hline Paroxysmal AF & $84(80.8 \%)$ & $47(83.9 \%)$ & $37(77.1 \%)$ & 0.377 \\
\hline Hypertension & $45(43.3 \%)$ & $22(39.3 \%)$ & $23(47.9 \%)$ & 0.376 \\
\hline Diabetes mellitus & $10(9.6 \%)$ & $7(12.5 \%)$ & $3(6.3 \%)$ & 0.335 \\
\hline Smoking & $5(4.8 \%)$ & $3(5.4 \%)$ & $2(4.2 \%)$ & 1.000 \\
\hline Heart failure & $13(12.5 \%)$ & $8(14.3 \%)$ & $5(10.4 \%)$ & 0.552 \\
\hline Dyslipidemia & $23(22.1 \%)$ & $12(21.4 \%)$ & $11(22.9 \%)$ & 0.855 \\
\hline Thyroid disorder & $11(10.6 \%)$ & $4(7.1 \%)$ & $7(14.6 \%)$ & 0.219 \\
\hline Previous myocardial infarction & $3(2.9 \%)$ & $1(1.8 \%)$ & $2(4.2 \%)$ & 0.594 \\
\hline Obesity (BMI > 30 Kg/m²) & $4(3.8 \%)$ & $2(3.6 \%)$ & $2(4.2 \%)$ & 1.000 \\
\hline Obstructive sleep apnea & $2(1.9 \%)$ & $1(1.8 \%)$ & $1(2.1 \%)$ & 1.000 \\
\hline Previous stroke & $1(0.9 \%)$ & $0(0 \%)$ & $1(2.1 \%)$ & 0.462 \\
\hline \multicolumn{5}{|c|}{ Medications } \\
\hline Beta-blockers & $64(61.5 \%)$ & $30(53.6 \%)$ & $34(70.8 \%)$ & 0.071 \\
\hline Antiarrhythmic & $63(60.6 \%)$ & $34(60.7 \%)$ & $29(60.4 \%)$ & 0.975 \\
\hline Anticoagulant & $88(84.6 \%)$ & $46(82.1 \%)$ & $42(87.5 \%)$ & 0.450 \\
\hline \multicolumn{5}{|c|}{ Additional characteristics } \\
\hline Periprocedural cardioversion & $33(31.7 \%)$ & $18(32.1 \%)$ & $15(31.3 \%)$ & 0.922 \\
\hline Sinus rhythm EKG & $55(52.9 \%)$ & $31(55.4 \%)$ & $24(50.0 \%)$ & 0.585 \\
\hline Diameter of the left atrium & $42.4 \pm 5.3$ & $43.0 \pm 5.3$ & $41.8 \pm 5.3$ & 0.277 \\
\hline Ejection fraction (\%) & $61.0 \pm 11.0$ & $60.0 \pm 12.0$ & $63.0 \pm 10.0$ & 0.098 \\
\hline Esophageal temperature & $37.3 \pm 1.0$ & $36.5 \pm 0.5$ & $38.3 \pm 0.5$ & $<0.001$ \\
\hline
\end{tabular}

ET max: maximum esophageal temperature; AF: atrial fibrillation; BMI: body mass index.

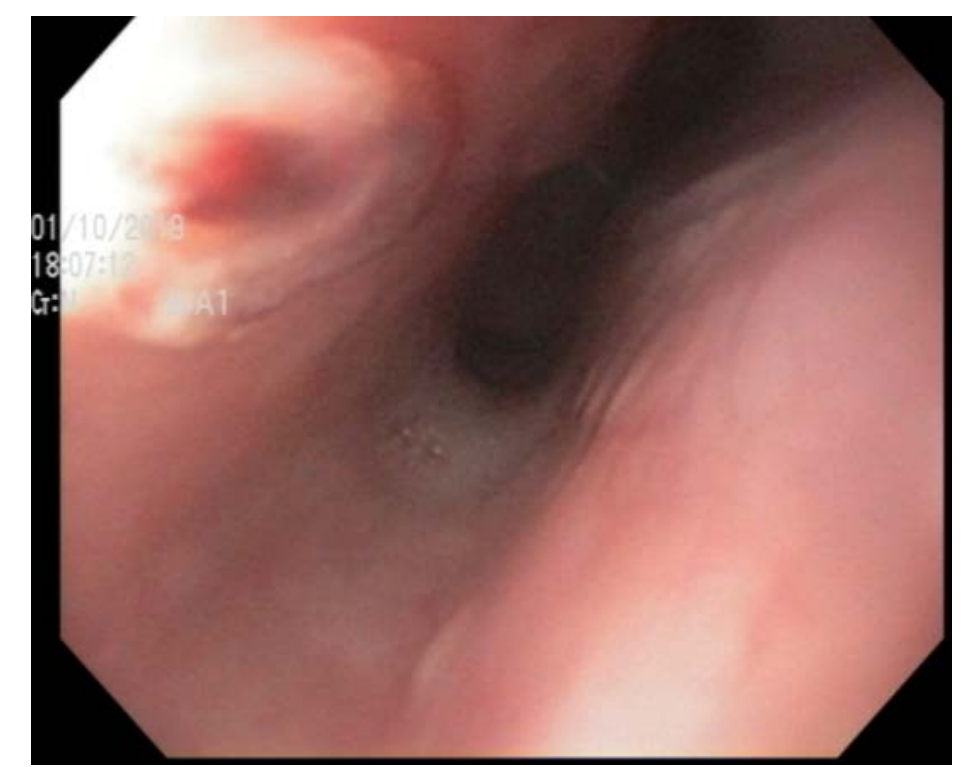

Figure 2. Esophageal lesion identified by post-procedure endoscopy. 
The AF recurrence rate found in the follow-up period was $9.6 \%$, corresponding to 10 patients. Of these, 3 patients (5.4\%) from the ET max group $<38^{\circ} \mathrm{C}$ and $7(14.6 \%)$ from the ET max group $\geq 38^{\circ} \mathrm{C}(\mathrm{p}=0.181)$. The detailed characteristics of the procedure and outcomes are described in Table 2. The multivariate analysis corroborated the results of the correlation by showing that esophageal temperature was not associated with $\mathrm{AF}$ recurrence after catheter ablation $(\mathrm{OR}=1.65,95 \%$ $\mathrm{CI}=0.84-3.24, \mathrm{p}=0.143)$.

Table 2. Procedure characteristics and outcomes.

\begin{tabular}{|c|c|c|c|c|}
\hline Characteristics and outcomes & $\begin{array}{c}\text { TOTAL } \\
(n=104)\end{array}$ & $\begin{array}{c}\mathrm{ET}<38^{\circ} \mathrm{C} \\
(\mathrm{n}=56)\end{array}$ & $\begin{array}{c}\mathrm{ET} \geq 38^{\circ} \mathrm{C} \\
(\mathrm{n}=48)\end{array}$ & $p$ \\
\hline Total electrical isolation of PV & $96(92.3 \%)$ & $53(94.6 \%)$ & $43(89.6 \%)$ & 0.466 \\
\hline Radioscopy time (min) & $20 \pm 14$ & $19 \pm 9$ & $20 \pm 18$ & 0.622 \\
\hline Procedure time (min) & $118 \pm 34$ & $115 \pm 32$ & $121 \pm 37$ & 0.429 \\
\hline Esophageal injury & $4(3.8 \%)$ & $2(3.6 \%)$ & $2(4.2 \%)$ & 1.000 \\
\hline AF recurrence & $10(9.6 \%)$ & $3(5.4 \%)$ & $7(14.6 \%)$ & 0.181 \\
\hline
\end{tabular}

PV: pulmonary vein; AF: atrial fibrillation; min: minutes.

\section{DISCUSSION}

It is important to identify high-risk population for esophageal injury during AF catheter ablation. In the Brazilian experience $^{6}$, one of the patients with AEF who died had the procedure performed by our team. This outcome motivated the development of an esophageal protection protocol to be applied to all $\mathrm{AF}$ ablation procedures, enabling further analysis.

Kapur et al. ${ }^{5}$ suggest an endoscopy within $72 \mathrm{~h}$ after the procedure if there is some technical factor during the procedure that lead to the risk of developing $\mathrm{AEF}$, such as extreme temperature rises/drops during ablation, postablation symptoms or preexisting esophageal pathology. If esophageal damage is not found on endoscopy, the patient has low risk for AEF. If there is esophageal ulceration, the patient has high risk, so they suggest proceeding with gastric acid suppression, bland diet, careful symptom monitoring and patient education.

Martinek et al. ${ }^{10}$ described an incidence of $2.2 \%$ of esophageal ulceration in 267 patients post-PVI, all with endoscopy within $24 \mathrm{~h}$ after procedure. They performed AF ablation with $25 \mathrm{~W}$ maximum energy at the left atrial posterior wall, without temperature monitoring. They found that a short distance between left atrial and esophagus was a predictor of esophageal ulceration.

In a single-center registry with 1802 patients post-AF ablation, 832 with endoscopic evaluation within 7 days after the procedure, the incidence of perforation or fistula was $0.6 \%$ (5/832 patients, 2 deaths). Thirty-five percent (285/832 patients) had esophageal temperature monitoring, and a maximum esophageal temperature above $40.5^{\circ} \mathrm{C}$ was predictor of $\operatorname{EDEL}(\mathrm{OR}=4.04,95 \% \mathrm{CI}=2.04-7.98, \mathrm{p}<0.001)$.

Regarding the esophageal temperature monitoring, Kadado et al. ${ }^{7}$ analyzed 7 studies with conflicting results. They concluded that the use of multipolar sensor probes appears to have higher sensitivity than single sensor probes to detect esophageal temperature rises. However, this has not translated into a lower incidence of esophageal injury. The authors advocate that operators should not rely on temperature sensing alone, but as part of a set of protective measures, such as avoiding areas directly contiguous to the esophagus when possible, reducing delivered energy and reducing the catheter contact force in the LA posterior wall.

In a systematic review, $\mathrm{Ha}$ et al. ${ }^{12}$ found no benefit of esophageal temperature monitoring, but the results were predominantly influenced by a study using RF nMARQ catheter. In this study with 145 patients, no using of esophageal temperature probe paradoxically reduced the incidence of esophageal termal damage (no use $0 \%$ vs. use $28 \%$, $\mathrm{p}<0.01$ ). A limitation with esophageal temperature monitoring is that it detects delayed temperature rises, which may not accurately reflect the intramural temperature. There remains no current consensus on an optimal luminal esophageal cut-off, as AEF has occurred at a recorded temperature as low as $36.4{ }^{\circ} \mathrm{C}^{14}$. 
This study was conducted with a specific prevention protocol using pre-, peri- and postprocedural measures. With regard to the maximum ET described, the cutoff value used was $38^{\circ} \mathrm{C}$. The current evidence about temperature limit do determine the interruption of the application is controversial. Some authors suggest maximum values between $39-41{ }^{\circ} \mathrm{C}$; however, the incidence of esophageal injuries varies from 10 to $47 \%$ using these cutoff values. Singh et al. ${ }^{15}$ showed a lower incidence of esophageal injury compared to not using a thermometer $6 \%$ vs. $36 \%$ ( $\mathrm{p}<0.006$ ), when a cutoff point of $38.5^{\circ} \mathrm{C}$ is used to interrupt the application of RF. The increase $\geq 1{ }^{\circ} \mathrm{C}$ in relation to the basal esophageal temperature can be used as a cut-off criteria; however, there is a report of the occurrence of esophageal injuries even with less heating. In addition, at each elevation of $1{ }^{\circ} \mathrm{C}$ in relation to the esophageal basal temperature, there is a $\mathrm{OR}=1.36$ for the development of thermal injury. In this study, trying to be more conservative, the $\mathrm{RF}$ applications were interrupted with a lower temperature $\left(38^{\circ} \mathrm{C}\right)$.

A meta-analysis of three studies ${ }^{8}$ showed beneficial effect of esophageal active cooling during AF ablation. Study protocols were based by injection 5-20 mL of ice-cold solution during RF energy delivery or when the luminal esophageal temperature reached a specific temperature. The results were quite consistent, with a $61 \%$ risk reduction to development of esophageal lesions grade III or IV (OR $=0.39,95 \% \mathrm{CI}=0.17-0.89)$. Grade III were defined by injuries $>3$ or with deeper layer, and grade IV were defined by presence of ulcer or clot. No patient was submitted to esophageal cooling in the protocol presented in this study.

Another AF ablation technique that has been used is the high-power short-duration (HPSD), that uses wide ranges of power $(35-50 \mathrm{~W})$ and duration $(2-20 \mathrm{~s})^{16}$. One of the theoretical advantages of HPSD ablation is that there may be more local heating of the tissue in the atrial myocardium and less distant collateral injuries to structures such as the esophagus. This technique was compared with the standard ablation in 687 patients whose esophageal involvement was assessed by magnetic resonance imaging (MRI) $24 \mathrm{~h}$ after the procedure, by late gadolinium enhancement (LGE). The HPSD ablation was performed with $50 \mathrm{~W}$ during $5 \mathrm{~s}$, and the standard ablation was performed with $<35 \mathrm{~W}$ at LA posterior wall during $10-30 \mathrm{~s}^{17}$. Esophageal LGE patterns were similar for HSDP vs. standard ablation (21\% vs. $28.3 \%$ mild, $11.5 \%$ vs. $11.5 \%$ moderate, and $2.8 \%$ vs. $2.7 \%$ severe; respectively, $\mathrm{p}=0,37$ ). There were no AEFs and the AF recurrence rates were also similar in a 2.5 -year follow-up period ( $42 \%$ vs. $41 \%, \mathrm{p}=0.57)$.

Currently, many groups have also performed AF ablation guided by prespecified ablation index (AI). An analysis of 211 patients undergoing first ablation using different AI target values (between 300 and 350 for LA posterior wall ablation) with endoscopic evaluation 1 to 3 days after procedure found that adopting an $\mathrm{AI} \leq 350$ was associated with 14\% esophageal thermal injury including $3 \%$ of severe esophageal thermal ulcers ${ }^{18}$. For patients included in the present study, HPSD or AI guided ablation were not used.

The recurrence rate was similar to that described in the literature. Analyzes are usually performed after 2-years period. The average follow-up of the studied patients was 1 year. In a previous study with an average follow-up of 373 days, the $\mathrm{AF}$ recurrence was $13.8 \%{ }^{19}$. In the $\mathrm{CABANA}$ trial recurrence assessment, the $\mathrm{AF}$ burden in 1 year in patients allocated to the ablation group was on average of $6.3 \%{ }^{20}$. The recurrence rate described in this analysis was $9.6 \%$.

This study has several limitations. First, it was an observational, nonrandomized and small-sized study. Confounding factors may be present and were not accounted. Although this study demonstrates a low incidence of esophageal injury, the true incidence is unknown. The end point of visible esophageal injury may underestimate the true incidence of intramural and nonapparent lesions. On the other hand, as no upper gastrointestinal endoscopy was performed prior do ablation, so it can only be suggested that the injuries found in 4 patients were related to RF energy. Patients undergoing cryoablation were excluded in this analysis, and recent techniques like high-power short-duration and ablation index guided ablation were not used. In addition, in the preventive method described, esophageal cooling was not performed. Regarding the assessment of AF recurrence, as the follow-up method was not equal for all patients, the recurrence rate may be underestimated. Similarly, the maintenance of antiarrhythmic drugs after procedure was defined individually, and this analysis was not performed.

As previously mentioned, there are many studies with conflicting results about esophageal temperature monitoring during AF ablation. This study, despite the limitations, described an apparently effective protocol, with a low incidence of esophageal injury and an acceptable AF recurrence rate, but further studies are needed to clarify this issue. 


\section{CONCLUSION}

The incidence of endoscopic asymptomatic esophageal lesions after AF catheter ablation with the use of a specific periprocedural esophageal protection protocol was low. There was no esophageal perforation. The AF recurrence rate was similar to that described in the literature.

\section{AUTHOR'S CONTRIBUTION}

Conceptualization: APB and CAK; Data Collection: Yanéz JPG, Andrade K, Boff CO and Ferreira FVC; Statistical analysis: Rösler ÁM; Investigation: Kalil CA, Bartholomay E, Borges AP and Gazzoni GF; Writing - Original Draft: Borges AP, Andrade K, Yanéz JPG and Boff CO; Writing - Review and Editing: Rösler ÁM, Lucchese FA and Kalil CA.

\section{REFERENCES}

1. Calkins H, Hindricks G, Cappato R, Kim Y-H, Saad EB, Aguinaga L, et al. 2017 HRS/EHRA/ECAS/APHRS/SOLAECE expert consensus statement on catheter and surgical ablation of atrial fibrillation: Executive summary. EP Europace. 2018;20(1):157-208. https://doi. org/10.1093/europace/eux275

2. Scanavacca MI, D’Ávila A, Parga J, Sosa E. Left atrial-esophageal fistula following radiofrequency catheter ablation of atrial fibrillation. J Cardiovasc Electrophysiol. 2004;15(8):960-2. https://doi.org/10.1046/j.1540-8167.2004.04083.x

3. Pappone C, Oral H, Santinelli V, Vicedomini G, Lang CC, Manguso F, et al. Atrio-esophageal fistula as a complication of percutaneous transcatheter ablation of atrial fibrillation. Circulation. 2004;109(22):2724-6. https://doi.org/10.1161/01.CIR.0000131866.44650.46

4. Kirchhof P, Benussi S, Kotecha D, Ahlsson A, Atar D, Casadei B, et al. 2016 ESC Guidelines for the management of atrial fibrillation developed in collaboration with EACTS. EP Europace. 2016;18(11):1609-78. https://doi.org/10.1093/europace/euw295

5. Kapur S, Barbhaiya C, Deneke T, Michaud GF. Esophageal injury and atrioesophageal fistula caused by ablation for atrial fibrillation. Circulation. 2017;136(13):1247-55. https://doi.org/10.1161/CIRCULATIONAHA.117.025827

6. De Vasconcelos JTM, Galvão Filho SS, Atié J, Maciel W, Souza OF, Saad EB, et al. Atrial-oesophageal fistula following percutaneous radiofrequency catheter ablation of atrial fibrillation: The risk still persists. EP Europace. 2017;19(2):250-8. https://doi.org/10.1093/ europace/euw284

7. Kadado AJ, Akar JG, Hummel JP. Luminal esophageal temperature monitoring to reduce esophageal thermal injury during catheter ablation for atrial fibrillation: A review. Trends Cardiovasc Med. 2019;29(5):264-71. https://doi.org/10.1016/j.tcm.2018.09.010

8. Leung LWM, Gallagher MM, Santangeli P, Tschabrunn C, Guerra JM, Campos B, et al. Esophageal cooling for protection during left atrial ablation: a systematic review and meta-analysis. J Interv Card Electrophysiol. 2020;59(2):347-55. https://doi.org/10.1007/ s10840-019-00661-5

9. Koneru JN, Kaszala K, Ellenbogen KA. Primum Non Nocere: The Paramount Importance of Esophageal Protection. Circ Arrhythmia Electrophysiol. 2017;10(8):e005614. https://doi.org/10.1161/CIRCEP.117.005614

10. Martinek M, Meyer C, Hassanein S, Aichinger J, Bencsik G, Schoefl R, et al. Identification of a high-risk population for esophageal injury during radiofrequency catheter ablation of atrial fibrillation: Procedural and anatomical considerations. Heart Rhythm. 2010;7(9):1224-30. https://doi.org/10.1016/j.hrthm.2010.02.027

11. Halbfass P, Pavlov B, Müller P, Nentwich K, Sonne K, Barth S, et al. Progression from esophageal thermal asymptomatic lesion to perforation complicating atrial fibrillation ablation: a single-center registry. Circ Arrhythmia Electrophysiol. 2017;10(8):e005233. https://doi.org/10.1161/CIRCEP.117.005233 
12. Ha FJ, Han H-C, Sanders P, Teh AW, O'Donnell D, Farouque O, et al. Prevalence and prevention of oesophageal injury during atrial fibrillation ablation: A systematic review and meta-analysis. EP Europace. 2019;21(1):80-90. https://doi.org/10.1093/europace/ euy 121

13. Deneke T, Müller P, Halbfaß P, Szöllösi A, Roos M, Krug J, et al. Effect of different ablation settings on acute complications using the novel irrigated multipolar radiofrequency ablation catheter (nMARQ). J Cardiovasc Electrophysiol. 2015;26(10):1063-8. https://doi. $\operatorname{org} / 10.1111 /$ jce.12736

14. Grubina R, Cha Y-M, Bell MR, Sinak LJ, Asirvatham SJ. Pneumopericardium following radiofrequency ablation for atrial fibrillation: Insights into the natural history of atrial esophageal fistula formation. J Cardiovasc Electrophysiol. 2010;21(9):1046-9. https://doi. org/10.1111/j.1540-8167.2010.01740.x

15. Singh SM, d'Avila A, Doshi SK, Brugge WR, Bedford RA, Mela T, et al. Esophageal injury and temperature monitoring during atrial fibrillation ablation. Circ Arrhythm Electrophysiol. 2008;1(3):162-8. https://doi.org/10.1161/CIRCEP.108.789552

16. Sauer WH, Tzou WS. With great power comes great responsibility: Defining the safety of high-power short-duration atrial ablation. Circ Arrhythm Electrophysiol. 2019;12(6):e007456. https://doi.org/10.1161/CIRCEP.119.007456

17. Baher A, Kheirkhahan M, Rechenmacher SJ, Marashly Q, Kholmovski EG, Siebermair J, et al. High-power radiofrequency catheter ablation of atrial fibrillation: using late gadolinium enhancement magnetic resonance imaging as a novel index of esophageal injury. JACC Clin Electrophysiol. 2018;4(12):1583-94. https://doi.org/10.1161/CIRCEP.119.007456

18. Halbfass P, Berkovitz A, Pavlov B, Sonne K, Nentwich K, Ene E, et al. Incidence of acute thermal esophageal injury after atrial fibrillation ablation guided by prespecified ablation index. J Cardiovasc Electrophysiol. 2019;30(11):2256-61. https://doi.org/10.1111/jce.14193

19. KochhÄuser S, Alipour P, Haig-Carter T, Trought K, Hache P, Khaykin Y, et al. Risk of stroke and recurrence after AF ablation in patients with an initial event-free period of 12 months. J Cardiovasc Electrophysiol. 2017;28(3):273-9. https://doi.org/10.1111/jce.13138

20. Poole JE, Bahnson TD, Monahan KH, Johnson G, Rostami H, Silverstein AP, et al. Recurrence of atrial fibrillation after catheter ablation or antiarrhythmic drug therapy in the CABANA Trial. J Am Coll Cardiol. 2020;75(25):3105-18. https://doi.org/10.1016/j. jacc.2020.04.065 